\title{
CONVERGENCE OF MEASURABLE RANDOM FUNCTIONS
}

\section{S. GRINBLAT}

\begin{abstract}
Using the theorem of Frechet and Kolmogorov about compact subsets of the space $L_{p}[0,1]$ and Prohorov's theorem about the convergence of measures defined on a complete metric space we proved in [2] the following theorem: Let $\xi_{1}(t), \ldots, \xi_{n}(t), \ldots$ and $\xi(t)$ be measurable random processes $(0<t<1)$ and suppose that there exist numbers $C$ and $p>1$ such that $E\left|\xi_{n}(t)\right|^{p}<C$ for all $n$ and $t$. If $E\left|\xi_{n}(t)\right|^{p} \rightarrow E|\xi(t)|^{p}$ for all $t$ and if for any finite set $\left\{t_{1}, \ldots, t_{k}\right\} \subset[0,1]$ the joint distribution of $\xi_{n}\left(t_{1}\right), \ldots, \xi_{n}\left(t_{k}\right)$ converges to the joint distribution of $\xi\left(t_{1}\right), \ldots, \xi\left(t_{k}\right)$, then the distribution of $f\left(\xi_{n}\right)$ converges to the distribution of $f(\xi)$ for any continuous functional $f$ on $L_{p}[0,1]$. In this paper this theorem is generalized to random measurable functions. The results of the present paper are related to the results of [1], [3], [4].
\end{abstract}

Let $(X, \rho)$ be a metric space. We call the set

$$
U_{y}^{r}=\{x \in X: \rho(y, x)<r\} \quad(r>0)
$$

a ball. We say that a set $U$ is a domain if $U$ is the union of a countable set of balls. We shall consider on $X$ only probability measures for which any ball is a measurable set. By $\rho_{y}(x)$ we denote the function $x \mapsto \rho(y, x)$.

LeMma. Let $\mu_{1}, \ldots, \mu_{n}, \ldots$, and $\mu$ be measures defined on $X$ such that for any finite set $\left\{y_{1}, \ldots, y_{k}\right\} \subset X$ the joint distribution of $\rho_{y_{1}}(x), \ldots, \rho_{y_{k}}(x)$ with respect to the measure $\mu_{n}$ converges to the joint distribution of $\rho_{y_{1}}(x), \ldots, \rho_{y_{k}}(x)$ with respect to the measure $\mu$. If $U$ is a domain and there exists a domain $V$ such that $U \cap V=\varnothing$ and $\mu(U)+\mu(V)=1$, then $\mu_{n}(U) \rightarrow \mu(U)$.

Proof. By definition $U=\bigcup_{i=1}^{\infty} U_{y_{i}}^{r_{i}}, V=\cup_{i=1}^{\infty} U_{z_{i}}^{s_{i}}$. Let $\varepsilon>0$. We will find numbers $k, d>0$ such that

$$
\begin{gathered}
\mu\left(U^{*} \cup V^{*}\right)>1-\varepsilon, \quad \text { where } U^{*}=\bigcup_{i=1}^{k} U_{y_{i}}^{r_{i}-d}, \quad V^{*}=\bigcup_{i=1}^{k} U_{z_{i}}^{s_{i}-d} ; \\
\mu\left(U^{*}\right)=\mu\left(\bar{U}^{*}\right), \quad \mu\left(V^{*}\right)=\mu\left(\bar{V}^{*}\right) .
\end{gathered}
$$

From the conditions of the lemma we obtain:

$$
\mu_{n}\left(U^{*}\right) \rightarrow \mu\left(U^{*}\right), \quad \mu_{n}\left(V^{*}\right) \rightarrow \mu\left(V^{*}\right) .
$$

Received by the editors March 15, 1978.

AMS (MOS) subject classifications (1970). Primary 60B05, 60B10.

Key words and phrases. Measurable random functions, convergence of joint distributions, convergence of measures. 
But $U^{*} \subset U, V^{*} \subset V, \mu(U)-\mu\left(U^{*}\right)<\varepsilon, \mu(V)-\mu\left(V^{*}\right)<\varepsilon, \mu(U)+\mu(V)$ $=1$. Since $\varepsilon$ is arbitrary we have $\mu_{n}(U) \rightarrow \mu(U)$.

Let $T=\{t\}$ be a space with a probability measure $\nu$, and $\Omega=\{\omega\}$ be a probability space with the probability $P$. If for any point $t \in T$ a random variable $\xi(t, \omega)$ is defined, then we say that $\xi(t, \omega)$ is a random function; if $\xi(t, \omega)$ is a measurable function on $T \times \Omega$ with respect to the measure $\nu \times P$, then we say that $\xi(t, \omega)$ is a measurable random function.

THEOREM. Let $\xi_{1}(t), \ldots, \xi_{n}(t), \ldots$ and $\xi(t)$ be measurable random functions defined on $(T, \nu)$, and suppose that there exists a function $f \in L_{p}(T, \nu), p \geqslant 1$, such that $E\left|\xi_{n}(t)\right|^{p}<f(t)$ for any $t$ and $n$. Suppose further that $E\left|\xi_{n}(t)\right|^{p} \rightarrow$ $E|\xi(t)|^{p}$ for any $t$ and that for any finite set $\left\{t_{1}, \ldots, t_{k}\right\} \subset T$ the joint distribution of $\xi_{n}\left(t_{1}\right), \ldots, \xi_{n}\left(t_{k}\right)$ converges to the joint distribution of $\xi\left(t_{1}\right), \ldots, \xi\left(t_{k}\right)$. Finally, let $\mu_{1}, \ldots, \mu_{n}, \ldots$ and $\mu$ be the measures on $L_{p}(T, \nu)$ defined by our random functions. If $U$ is a domain in $L_{p}(T, \nu)$ and there exists a domain $V \subset L_{p}(T, \nu)$ such that $U \cap V=\varnothing$ and $\mu(U)+\mu(V)=1$, then $\mu_{n}(U) \rightarrow \mu(U)$.

Before proving this theorem we point out an important proposition which it implies. Suppose that there exists a separable space $Z \subset L_{p}(T, \nu)$ such that $\mu(Z)=1$; under this assumption, any open subset of $L_{p}(T, \nu)$ is measurable with respect to $\mu$. Let $U$ be an open subset of $L_{p}(T, \nu)$ such that $\mu(U)=\mu(\bar{U})$. Then $\bar{\mu}_{n}(U) \rightarrow \mu(U), \underline{\mu}_{n}(U) \rightarrow \mu(U)$, where $\bar{\mu}_{n}(U)$ and $\underline{\mu}_{n}(U)$ denote the outer and inner measures of $\bar{U}$.

Proof of The Theorem. Let $\left\{y_{1}(t), \ldots, y_{k}(t)\right\} \subset L_{p}(T, \nu)$. In view of the lemma it is enough to prove that the joint distribution of

$$
\int_{T}\left|y_{1}(t)-\xi_{n}(t)\right|^{p} d \nu, \ldots, \int_{T}\left|y_{k}(t)-\xi_{n}(t)\right|^{p} d \nu
$$

converges to the joint distribution of

$$
\int_{T}\left|y_{1}(t)-\xi(t)\right|^{p} d \nu, \ldots, \int_{T}\left|y_{k}(t)-\xi(t)\right|^{p} d \nu
$$

Let $\chi(\omega)$ be a random variable. For any $s>0$ we set

$$
\chi^{(s)}(\omega)= \begin{cases}0 & \text { if }|\chi(\omega)|<s \\ |\chi(\omega)|^{p} & \text { if }|\chi(\omega)| \geqslant s\end{cases}
$$

We fix $\varepsilon>0$. There exist a natural number $N$, a number $r>0$ and a measurable set $Q \subset T$ such that

(a) $E \eta_{n i}^{(r)}(t)<\varepsilon$ if $n \geqslant N, t \in Q$ and where $\eta_{n i}=y_{i}(t)-\xi_{n}(t)$;

(b) $\int_{T \backslash Q}\left|y_{i}(t)\right|^{p} d \nu<\varepsilon, 1 \leqslant i \leqslant k$;

(c) $\int_{T \backslash Q}|f(t)|^{p} d \nu<\varepsilon$.

For any $s>0$ consider the real function $\varphi_{s}(x)=\min \left(|x|^{p}, s^{p}\right)$. It is obvious 
that for $n \geqslant N$,

$$
\begin{aligned}
E \int_{T} \mid y_{i}(t)- & \left.\xi_{n}(t)\right|^{p} d \nu-E \int_{T} \varphi_{r}\left(y_{i}(t)-\xi_{n}(t)\right) d \nu \\
& \leqslant E \int_{T \backslash Q}\left|y_{i}(t)-\xi_{n}(t)\right|^{p} d \nu+\int_{Q} E \eta_{n, i}^{(r)}(t) d \nu \\
& \leqslant 2^{p} \int_{T \backslash Q}\left|y_{i}(t)\right|^{p} d \nu+2^{p} \int_{T \backslash Q}\left|\xi_{n}(t)\right|^{p} d \nu+\varepsilon \\
& \leqslant 2^{p} \cdot \varepsilon+2^{p} \int_{T \backslash Q}|f(t)|^{p} d \nu+\varepsilon \leqslant \varepsilon\left(2^{p+1}+1\right) .
\end{aligned}
$$

If $\varepsilon$ is small enough, then for $n \geqslant N$ the joint distribution of (1) differs little from the joint distribution of

$$
\int_{T} \varphi_{r}\left(y_{1}(t)-\xi_{n}(t)\right) d \nu, \ldots, \int_{T} \varphi_{r}\left(y_{k}(t)-\xi_{n}(t)\right) d \nu,
$$

and the joint distribution of (2) differs little from the joint distribution of

$$
\int_{T} \varphi_{r}\left(y_{1}(t)-\xi(t)\right) d \nu, \ldots, \int_{T} \varphi_{r}\left(y_{k}(t)-\xi(t)\right) d \nu .
$$

Therefore it is enough to prove that the joint distribution of (3) converges to the joint distribution of (4). Let $m_{1}, \ldots, m_{k}$ be any set of $k$ natural numbers. We have

$$
\begin{aligned}
& E \prod_{i=1}^{k}\left(\int_{T} \varphi_{r}\left(y_{i}(t)-\xi_{n}(t)\right) d \nu\right)^{m_{i}} \\
& \quad=\int_{T^{*}} E \prod_{i=1}^{k} \prod_{j=1}^{m_{i}} \varphi_{r}\left(y_{i}\left(t_{i j}\right)-\xi_{n}\left(t_{i j}\right)\right) d \nu^{*} \\
& \quad \rightarrow \int_{T^{*}} E \prod_{i=1}^{k} \prod_{j=1}^{m_{i}} \varphi_{r}\left(y_{i}\left(t_{i j}\right)-\xi\left(t_{i j}\right)\right) d \nu^{*} \\
& \quad=E \prod_{i=1}\left(\int_{T} \varphi_{r}\left(y_{i}(t)-\xi(t)\right) d \nu\right)^{m_{i}},
\end{aligned}
$$

where $T^{*}$ is the product of $\sum_{i=1}^{k} m_{i}$ copies of the space $T$ and the measure $\nu^{*}$ is the product of $\sum_{i=1}^{k} m_{i}$ measures $\nu$. From this it follows immediately that the joint distribution of (3) converges to the joint distribution of (4).

In classical measure theory the basic theorem is the following:

THEOREM OF LeBesGue. Let $\left\{\varphi_{n}(t)\right\} \subset L_{1}(T, v), \varphi_{n}(t) \rightarrow \varphi(t)$ for all $t$, and suppose there exists $f \in L_{1}(T, v)$ such that $\left|\varphi_{n}(t)\right| \leqslant f(t)$ for all $t$. Then $\varphi(t) \in L_{1}(T, \nu)$ and $\left\|\varphi_{n}-\varphi\right\| \rightarrow 0$.

It is possible to say that the theorem proved in this paper is a resasonable infinite generalization of the theorem of Lebesgue. 


\section{REFERENCES}

1. L. S. Grinblat, Compactifications of spaces of functions and integration of functionals, Trans. Amer. Math. Soc. 217 (1976), 195-223.

2. Math. Soc. 61 (1976), 371-376.

3. Convergence of random processes without discontinuities of the second kind and limit theorems for sums of independent random variables, Trans. Amer. Math. Soc. 234 (1977), 361-379. 4. appear).

Department of Mathematics, Bar-Ilan University, Ramat-Gan, IsRael 